\title{
PREVALENCIA DE FACTORES DE RIESGO CARDIOVASCULAR Y RIESGO METABÓLICO EN ESCOLARES, UNIVERSITARIOS Y MUJERES DE ORGANIZACIONES SOCIALES DE BASE EN DISTRITOS DE LIMA, CALLAO, LA LIBERTAD Y AREQUIPA, PERÚ 2011
}

\author{
Eloísa Núñez-Robles ${ }^{1, a}$, Cleopatra Huapaya-Pizarro" ${ }^{1, b}$, Rogger Torres-Lao ${ }^{1, c}$, Silvia Esquivel-León ${ }^{1, b}$, \\ Víctor Suarez-Moreno ${ }^{2, d}$, Myriam Yasuda-Espinoza ${ }^{2, e}, G^{2}$ Giovanna Sanjinés-López z2,f.
}

RESUMEN

Objetivos. Determinar la prevalencia de hipertensión arterial y factores de riesgo cardiovascular y riesgo metabólico (FRCRM) en escolares, universitarios y mujeres de organizaciones sociales de base (OSB) de cuatro distritos en Perú. Materiales y métodos. Estudio transversal analítico realizado el 2011 en distritos de Lima, Callao, La Libertad y Arequipa, con representatividad para escolares, universitarios y mujeres de OSB. Se realizó una encuesta, mediciones antropométricas, medición de presión arterial y toma de muestra sanguínea en ayunas para determinar el nivel de glucosa y lípidos; calculando prevalencias de exceso de peso, hipertensión, hipercolesterolemia, sospecha de diabetes, entre otras. La variable resultado principal fue la prevalencia de sobrepeso. Resultados. Se incluyó 1127 personas, 283 (25,1\%) escolares, 431 (38,3\%) universitarios y $413(36,6 \%)$ mujeres de las OSB, las tasas de no respuesta fueron de 3; 0 y $8 \%$ respectivamente; las edades promedio en los tres grupos fueron $14,3 \pm 0,9 ; 19,6 \pm 2,8$; y 46,1 $\pm 15,3$ años respectivamente. La prevalencia de sobrepeso/obesidad (IMC $\geq 25$ ) fue de 11,$3 ; 20,9$ y $73,4 \%$ en cada grupo; de hipertensión fue de 0,$7 ; 1,2$, y $12,8 \%$ respectivamente; de hipercolesterolemia ( $\geq 200 \mathrm{mg} / \mathrm{dL}$ ) fue de 5,$2 ; 11,5$ y $50,1 \%$ en cada grupo; y de sospecha de diabetes fue de 1,$4 ; 1,0$ y $20,3 \%$ en los grupos de escolares, universitarios y mujeres de OSB. Conclusiones. La prevalencia de los FRCRM, en general, se incrementa en los grupos de mayor edad; en los grupos de escolares y universitarios son más frecuentes los problemas de sobrepeso y obesidad, en particular la obesidad abdominal.

Palabras clave: Hipertensión; Diabetes mellitus; Dislipidemias; Obesidad; Enfermedades cardiovasculares (fuente: DeCS BIREME).

\section{PREVALENCE OF CARDIOVASCULAR AND METABOLIC RISK FACTORS IN SCHOOL STUDENTS, UNIVERSITY STUDENTS, AND WOMEN FROM COMMUNITY-BASED ORGANIZATIONS IN THE DISTRICTS OF LIMA, CALLAO, LA LIBERTAD AND AREQUIPA, PERU 2011}

\begin{abstract}
Objectives. To determine the prevalence of arterial hypertension, and cardiovascular and metabolic risk factors in school students, college students and women from community-based organizations (CBO) in four districts in Peru. Materials and methods. Cross-sectional study conducted in 2011 in the districts of Lima, Callao, La Libertad and Arequipa with school students, university students and women from CBOs. A survey, anthropometric measurements, blood pressure measurements and fasting blood sampling were conducted to determine glucose and lipid levels. Thus, prevalence of overweight, hypertension, hypercholesterolemia, suspected diabetes, and other variables, were calculated. The main outcome variable was prevalence of overweight. Results. 1,127 people were included: $283(25.1 \%)$ school students, 431 $(38.3 \%)$ university students, and 413 women $(36.6 \%)$ from CBOs. Non-response rates were $3 \%, 0 \%$ and $8 \%$, respectively. The average ages in the three groups were $14.3 \pm 0.9 ; 19.6 \pm 2.8$; and $46.1 \pm 15.3$ years, respectively. Prevalence of overweight/obesity (BMI $\geq 25$ ) was $11.3 \%, 20.9 \%$, and $73.4 \%$; hypertension was $0.7 \% ; 1.2 \%$, and $12.8 \%$; high cholesterol ( $\geq 200 \mathrm{mg} / \mathrm{dL}$ ) was $5.2 \%, 11.5 \%$, and $50.1 \%$; and suspected diabetes was $1.4 \% ; 1.0 \%$, and $20.3 \%$ respectively in each group of school students, university students and women from CBOs. Conclusions. The prevalence of cardiovascular and metabolic risk factors generally increased in older age groups. In school and university aged groups, the most frequent problems were overweight and obesity, particularly abdominal obesity.
\end{abstract}

Key words: Hypertension; Diabetes mellitus; Dyslipidemias; Obesity; Cardiovascular diseases (source: MeSH, NLM).

\footnotetext{
Agencia Adventista para el Desarrollo y Recursos Asistenciales (ADRA Perú). Lima, Perú.

Instituto Nacional de Salud. Lima, Perú.

Licenciada en Enfermería; ${ }^{b}$ licenciada en Enfermería, magíster en Salud Pública; ${ }^{\mathrm{c}}$ médico epidemiólogo; ${ }^{\mathrm{d}}$ médico infectólogo; ${ }^{\mathrm{e}}$ médico patólogo clínico; tecnólogo médico.

Recibido: : 12-06-14 Aprobado: 05-11-14

Citar como: Núñez Robles E, Huapaya Pizarro C, Torres R, Esquivel-León S, Suarez Moreno S, Yasuda Espinoza MB, et al. Prevalencia de enfermedades de riesgo cardiovascular y riesgo metabólico en escolares, universitarios y mujeres de organizaciones sociales de base en distritos de Lima, Callao, La Libertad y
} Arequipa, Perú 2011. Rev Peru Med Exp Salud Publica. 2014;31(4):652-9. 


\section{INTRODUCCIÓN}

Los factores de riesgo cardiovascular y metabólico (FRCRM) incluyen la hipertensión, la diabetes, las dislipidemias, la obesidad, entre otros; actualmente, desencadenan las principales causas de muerte en el mundo y en países de ingresos medios o bajos (1), como las muertes por diabetes mellitus, enfermedades hipertensivas y tumores malignos. En el Perú, debido a la transición epidemiológica evidenciada desde inicios del nuevo milenio (2), los FRCRM representan la mayor cantidad de causas de muerte (3) y años de vida saludables perdidos (4), superando a las enfermedades infecciosas y a los accidentes de tránsito, con una tendencia anual creciente ${ }^{(5)}$. Ello se debe a que los FRCRM tienen una mayor duración, pueden prolongarse de por vida, y producir mayor discapacidad.

La razón del incremento de FRCRM se debe al envejecimiento poblacional, dietas menos saludables y a la menor actividad física debido a influencias tecnológicas y culturales. En el contexto peruano, un aspecto importante se debe a la migración urbano-rural, pues se conservan los hábitos alimentarios rurales, adecuados para gastos metabólicos altos, a pesar que la actividad física en el área urbana es menor ${ }^{(6)}$. Por ejemplo, en Perú, el exceso de peso (sobrepeso y obesidad) tiene una prevalencia más alta en la población adulta joven $(63,2 \%)^{(7)}$; sin embargo, en áreas urbanas su prevalencia es mayor al $30 \%$ tanto en niños como adolescentes ${ }^{(7)}$. Otros estudios indican que, además de la obesidad, la presencia de síndrome metabólico es cada vez más frecuente, llegando a un 8,8\% entre adolescentes ${ }^{(8)}$. En comparación con otros FRCRM, como la hipertensión arterial, que está presente en un $1,5 \%$ de adolescentes de 12 a 17 años ${ }^{(9)}$. Esto nos indica que existe tendencia al desarrollo de FRCRM desde poblaciones muy jóvenes, aun cuando sus prevalencias sean bajas.

En este escenario, el Ministerio de Salud de Perú, a través de la Estrategia Sanitaria Nacional de Enfermedades No Transmisibles, viene emitiendo políticas que priorizan el fortalecimiento y el impulso de estrategias, planes y acciones del sector, con el propósito de mejorar la salud y la calidad de vida de la población peruana en distintas etapas de la vida (10). Promoviendo una vida sana, con comportamientos saludables, salud mental y construcción de entornos saludables (11); debido a que existe tendencia al desarrollo de FRCRM que son potencialmente modificables, como los estilos de vida o hábitos alimentarios y actividad física, que al ser incorporados desde la niñez pueden prevenir el desarrollo de FRCRM (12).
Es así que ADRA Perú planteó la implementación del proyecto de intervención denominado "Adelante: Estrategia preventiva promocional para disminuir la incidencia de enfermedades no transmisibles en adolescentes, jóvenes y adultos en instituciones educativas, universidades y organizaciones sociales de base de cuatro distritos de la zona urbana del Perú". Esta estrategia ha sido adoptada debido a que hay evidencia que indica que es más fácil y menos costoso ayudar a que una persona se mantenga saludable, a tener que curarla de una enfermedad. Esta intervención centró su esfuerzo en el cambio de estilos de vida para mantenerse saludable y prevenir enfermedades y, luego de 3 años de intervención, espera disminuir en 10\% (sobre la línea) la prevalencia de sobrepeso y otros factores de riesgo de enfermedades no transmisibles (ENT) en escolares, universitarios y adultos participantes. Considerando la importancia de los esfuerzos en la generación de evidencias, el Instituto Nacional de Salud, ha reconocido la importancia de esta intervención y ha decidido coparticipar, sumar fortalezas técnicas y trabajar en todos los procesos de planificación, ejecución y publicación de este estudio de línea de base.

\section{MATERIALES Y MÉTODOS}

Se realizó un estudio transversal analítico que incluyó a adolescentes, jóvenes y mujeres adultas. El estudio se desarrolló durante el año 2011 en el distrito de Trujillo, departamento de La Libertad, ubicado al norte del Perú; en el distrito de Hunter, departamento de Arequipa, ubicada al sur; y en los distritos de Chaclacayo, departamento de Lima, y Bellavista, provincia constitucional del Callao, ubicados en la costa centro. Este estudio representa la línea basal del proyecto ADELANTE ${ }^{(13)}$, que tenía como objetivo disminuir la incidencia de FRCRM en adolescentes, jóvenes y mujeres adultas a través de la promoción de estilos de vida saludables.

\section{POBLACIÓN Y MUESTRA}

Se estudió a tres poblaciones 1) escolares, que incluye adolescentes del segundo y tercer año de educación secundaria; 2) universitarios, conformado por adolescentes y jóvenes del primer y segundo año de educación superior universitaria, y 3) mujeres de organizaciones sociales de base (OSB), que incluye mujeres de 18 años a más, usuarias de comedores populares y comités de vaso de leche. En estos grupos, se excluyeron aquellos que tuvieran enfermedad aguda con descanso médico, las mujeres embarazadas o las personas con discapacidades físicas, ya que las mediciones antropométricas podrían estar sesgadas con los equipos utilizados. 
Debido a que esta medición sirvió como línea de base de la intervención, la muestra fue diseñada con representatividad para cada grupo en cada ciudad. Para el cálculo del tamaño de muestra se consideró la reducción del sobrepeso/obesidad en $10 \%$ en las poblaciones intervenidas dado que es el principal indicador de impacto del proyecto ADELANTE. El cálculo de tamaño de muestra se realizó empleando la fórmula para diferencia de proporciones. Considerando el objetivo de la intervención, la variable de referencia para calcular la muestra fue la prevalencia de sobrepeso (outcome principal) $22,4 \%$ en escolares, $35,0 \%$ en universitarios y $46,6 \%$ en mujeres adultas, datos obtenidos de estudios en poblaciones similares en San Salvador de Jujuy Argentina( $^{(14)}$; en Talca - VII Región del Maule, Chile ${ }^{(15)}$, y en Perú, Lima Metropolitana, resto de costa, sierra urbana, sierra rural y selva ${ }^{(16)}$, respectivamente. Para el cálculo de la muestra se consideró un nivel de confianza de 95\% y un margen de error del $5 \%$, siendo representativa para las tres poblaciones indicadas. Además, se consideró que la tasa de no respuesta sería de $20 \%$ para escolares y $40 \%$ para universitarios y mujeres de OSB.

Las muestras fueron representativas para cada población y para cada lugar; el tamaño de muestra en cada escuela fue 73; la sumatoria de las cuatro escuelas 292; la muestra en universitarios fue 106, y la suma 424; la de mujeres de OSB fue 113, y la suma 452. La intervención fue planificada para ser desarrollada en estos cuatro distritos específicos y en las siguientes instituciones (Tabla 1).

La selección de los elementos muestrales fue sistemática con arranque aleatorio, para ello se empleó un listado de alumnos de las instituciones educativas, de las universidades y las usuarias de OSB.

\section{RECOLECCIÓN Y PROCESAMIENTO DE DATOS}

Las personas seleccionadas respondieron un cuestionario estructurado que incluía las variables descritas en el siguiente apartado. Además, se les realizó mediciones antropométricas (peso, talla, perímetro de cintura) y de la presión arterial; al día siguiente de la encuesta, estando en ayunas, se les realizó la toma de muestra venosa para la evaluación de lípidos y glucosa.

La evaluación de la presión arterial se realizó con un tensiómetro de columna de mercurio y estetoscopio, en tres oportunidades el mismo día de la encuesta; se consideró el promedio de las tres mediciones de presión arterial.

Respecto a las muestras venosas, se tomaron dos muestras de sangre por a cada persona: un tubo de $10 \mathrm{~mL}$ sin anticoagulante para obtener el suero y un tubo de 2 $\mathrm{mL}$ con fluoruro para preservar los niveles de glucosa. Las muestras fueron trasladadas a los laboratorios de referencia del INS más cercanos, en un tiempo no superior a 1 hora, en donde fueron centrifugadas y se separó el suero, el cual fue refrigerado respetando la cadena de frio. En el caso de los distritos de Bellavista y Chaclacayo, los sueros fueron enviados el mismo día de la obtención, y en el caso de los distritos alejados como Hunter y Trujillo, los sueros fueron congelados con hielo seco y enviados al INS al día siguiente de su obtención.

Los datos obtenidos pasaron por el control de calidad de los coordinadores y luego fueron ingresados a una base de datos en Epiinfo 2000. El control de calidad de la digitación se realizó mediante la revisión de las frecuencias de todas las variables y el contraste del $10 \%$ de encuestas. Tanto las encuestas y las fichas de datos de las muestras fueron codificadas con un código único de barras, el cual permitió añadir los resultados de laboratorio a la base de datos de encuestas.

\section{VARIABLES}

\section{Marcadores diagnósticos de enfermedades no transmisibles}

Hipertensión arterial: la presión arterial se clasificó según el Seventh Report of the Joint National Committee (17), solo se consideró las categorías hipertenso y normotenso (PAS $\geq 140$ y $P A D \geq 90$ )

Tabla 1. Poblaciones estudiadas

\begin{tabular}{|c|c|c|c|}
\hline Distritos & Comedores Populares & $\begin{array}{c}\text { Institución educativa } \\
\text { (alumnos del } 2 .^{\circ} \text { y } 3 .^{\text {er }} \text { año de secundaria, } \\
\text { entre } 14 \text { y } 15 \text { años) }\end{array}$ & $\begin{array}{l}\text { Universidad (alumnos del 1er y } \\
\text { 2do año, entre } 17 \text { y } 24 \text { años) }\end{array}$ \\
\hline Trujillo & $\begin{array}{l}\text { Comedores populares del } \\
\text { distrito de Trujillo }\end{array}$ & I.E. N. ${ }^{\circ} 8010$ Ricardo Palma del distrito de Trujillo & $\begin{array}{l}\text { Universidad Nacional de Trujillo del } \\
\text { distrito de Trujillo }\end{array}$ \\
\hline Arequipa & $\begin{array}{l}\text { Comedores populares de los } \\
\text { distritos de Hunter }\end{array}$ & $\begin{array}{l}\text { I.E. Juan Pablo Vizcardo Guzmán del distrito de } \\
\text { Arequipa }\end{array}$ & $\begin{array}{l}\text { Universidad Nacional San Agustín } \\
\text { del distrito de Arequipa }\end{array}$ \\
\hline Lima & $\begin{array}{l}\text { Comedores populares del } \\
\text { distrito de Chaclacayo }\end{array}$ & $\begin{array}{l}\text { I.E. Ramón Castilla } \\
\text { del distrito de Chaclacayo }\end{array}$ & $\begin{array}{l}\text { Universidad Peruana Unión del } \\
\text { distrito de Chaclacayo }\end{array}$ \\
\hline
\end{tabular}


Sobrepeso/Obesidad: se definió según la clasificación de la OMS, considerando como sobrepeso el índice de masa corporal (IMC) de $25 \mathrm{a}<30 \mathrm{~kg} / \mathrm{m}^{2}$, y obesidad si el IMC fue $\geq 30 \mathrm{~kg} / \mathrm{m}^{2(18)}$.

Obesidad abdominal: definido como $\geq 94 \mathrm{~cm}$ en varones $y \geq 80$ en mujeres ${ }^{(19)}$.

Sospecha de diabetes: glucosa en sangre en ayunas mayor a $126 \mathrm{mg} / \mathrm{dL}$ en una sola toma. No se completó el criterio de dos tomas para el diagnóstico certero de diabetes ${ }^{(20)}$.

Hipercolesterolemia: se consideró la clasificación del Adult Treatment Panel (ATP III) para considerar valores elevados de colesterol total si era $\geq 200 \mathrm{mg} / \mathrm{dL}$, de LDL si era $\geq 130 \mathrm{mg} / \mathrm{dL}$ y se consideró HDL bajo si era $<40 \mathrm{mg} / \mathrm{dL}^{(21)}$.

Hipertrigliceridemia: definida como triglicéridos $\geq 150 \mathrm{mg} / \mathrm{dL}^{(21)}$.

\section{Prácticas saludables}

La intervención del proyecto ADELANTE (Agua pura, Descanso, Ejercicio físico, Luz del Sol, Aire puro, Nutrición adecuada, Temperancia o manejo adecuado del estrés y Esperanza) busca la realización de al menos dos de las siguientes prácticas saludables:

Consumo de agua apropiado: ingesta de al menos 5 vasos de agua pura por día.

Descanso: sueño entre 7 a 8 horas cada noche

Ejercicio físico: al menos 30 minutos de actividad física moderada o intensa, cinco veces a la semana.

Nutrición adecuada: consumo de cinco porciones de frutas o verduras cada día.

Manejo adecuado del estrés: adecuado afrontamiento de conflictos considerando las reacciones más usuales ante problemas y conflictos cotidianos. Se consideró la clasificación y escala empleada en la Encuesta Nacional de Calidad de Vida y Salud de Chile ${ }^{(22)}$; donde se formaron cuatro categorías: 1) autodestructiva: fuma o come o se sirve un trago; 2) evasiva: busca estar solo o duerme o no hace nada; 3) hostil: Le grita a la primera persona que lo hace enojar; 4) activa: sale o ve televisión o escucha música o lee o juega con el computador. Se considera como adecuada una conducta "activa" ante un conflicto.

\section{Prácticas nocivas y otras variables}

Consumo de cigarrillos. Si alguna vez en la vida fumó; si fuma en reuniones o fiestas.

Consumo de licor. Si alguna vez ha consumido licor; ha consumido durante el último mes o la última semana.

Sexo, edad, distrito de procedencia. Aseguramiento en un sistema de salud.

\section{ANÁLISIS ESTADÍSTICO}

Las variables categóricas se presentan con frecuencias absolutas y relativas, las cuantitativas en medias \pm desviación estándar. Las prevalencia se presentan además con sus respectivos intervalos de confianza al $95 \%$. Se compararon los promedios entre grupos empleando la prueba de ANOVA; en el caso de los porcentajes, se compararon empleando chi cuadrado. Todos los resultados en tablas se muestran desagregadas por los grupos poblacionales evaluados (escolares, universitarios y mujeres de OSB). Se consideraron como significativos los valores $p<0,05$. Los datos obtenidos se analizaron usando el programa Stata v.12.1.

\section{CONSIDERACIONES ÉTICAS}

Todas las personas fueron informadas sobre los alcances del proyecto, sus beneficios y riesgos. La aceptación de participación en el estudio fue voluntaria, previa firma del consentimiento; en el caso de los menores de edad, el consentimiento fue firmado por los padres o apoderados. Los participantes a los cuales se les diagnosticó algún daño o factor de riesgo, fueron derivados al establecimiento de salud y se les incluyó en el programa de prevención del proyecto ADELANTE para su supervisión. Se contó con la aprobación del Comité de Investigación y Comité de Ética del Instituto Nacional de Salud.

\section{RESULTADOS}

Se analizó un total de 1127 personas, 25,1\% escolares, $38,3 \%$ universitarios y $36,6 \%$ mujeres de OSB. El $81,2 \%$ del total de la población fueron mujeres. El rendimiento de la muestras fue, en promedio, $97 \%$ en escolares, $100 \%$ en universitarios y $92 \%$ en OSB; en este sentido, las tasas de no respuesta fueron de 3; 0 y $8 \%$ respectivamente. Las pérdidas no fueron reemplazadas ya que la muestra fue suficiente considerando la no respuesta.

Las características de las poblaciones se describen en la Tabla 2. Las edades en los escolares y universitarios fueron similares en todos los distritos, mientras que en las mujeres de OSB, hubo mayor variación, siendo estas edades significativamente distintas $(p<0,001)$.

Entre los escolares y universitarios no se encontraron diferencias significativas $(p>0,05)$ al comparar el peso, talla, IMC y perímetro de cintura entre los distritos de procedencia. En cambio, en las mujeres de OSB se identificaron diferencias en el peso $(p<0,001)$, IMC $(p<0,01)$ 
Tabla 2. Características de la población incluida

\begin{tabular}{lcccc}
\hline & Escolares & Universitarios & Madres OSB & Total \\
\cline { 2 - 5 } & $\mathbf{n = 2 8 3}$ & $\mathbf{n = 4 3 1}$ & $\mathbf{n = 4 1 3}$ & $\mathbf{n = 1 1 2 7}$ \\
\hline Sexo femenino $(\%)$ & 52,7 & 81,9 & 100 & 81,2 \\
Edad (años) & $14,3 \pm 0,9$ & $19,6 \pm 2,8$ & $46,1 \pm 15,3$ & $27,9 \pm 16,8$ \\
Peso $(\mathrm{kg})^{*}$ & $51,7 \pm 9,2$ & $56,7 \pm 8,8$ & $64,7 \pm 11,9$ & $58,4 \pm 11,3$ \\
Talla $(\mathrm{cm})^{\star}$ & $155,7 \pm 7,5$ & $156,9 \pm 7,0$ & $151,1 \pm 6,5$ & $154,5 \pm 7,4$ \\
Índice de masa corporal $\left(\mathrm{kg} / \mathrm{m}^{2}\right)^{*}$ & $21,3 \pm 3,1$ & $23,0 \pm 3,1$ & $28,3 \pm 4,9$ & $24,5 \pm 4,9$ \\
Perímetro de cintura $(\mathrm{cm})^{*}$ & $75,7 \pm 8,0$ & $79,4 \pm 9,4$ & $93,9 \pm 12,1$ & $83,8 \pm 12,9$ \\
Acceso a un seguro médico $(\%)$ & 64,3 & 47,7 & 58,9 & 55,9 \\
\hline
\end{tabular}

* media \pm desviación estándar

y perímetro de cintura $(p=0,01)$, mas no en la talla $(p=0,82)$. Las madres OSB del distrito de Trujillo-La Libertad fueron las que tuvieron menor peso $(61,8 \pm 10,4 \mathrm{Kg})$, mientras que las de Bellavista-Callao fueron las que tuvieron el mayor peso promedio $(68,4 \pm 13,6 \mathrm{Kg})$; estas diferencias se mantuvieron para el IMC y perímetro de cintura.

\section{MARCADORESDIAGNÓSTICOSDEENFERMEDADES CARDIOVASCULARES Y METABÓLICAS}

Las prevalencias de hipercolesterolemia total y obesidad abdominal incrementaron en frecuencia desde los escolares, universitarios hasta las mujeres de OSB (chi cuadrado tendencia $p<0,001$ ), la hipertensión en universitarios y mujeres de OSB tuvieron el mismo comportamiento. Los escolares tuvieron un alto porcentaje de HDL bajo, sobrepeso u obesidad abdominal (14,1\%). En el caso de los universitarios, el porcentaje de obesidad abdominal fue del 35,0\%; mientras que en las mujeres de OSB el $91,8 \%$ presentó obesidad abdominal.

En el grupo de escolares, el factor de riesgo con mayor prevalencia fue el colesterol HDL bajo, seguido de obesidad abdominal, las demás prevalencias de FRCRM se muestran en la Tabla 3. No hubo diferencias en los porcentajes de sospecha de diabetes ni hipertensión arterial al compararlos entre distritos. Sin embargo, en el distrito de Hunter-Arequipa se identificó el mayor porcentaje de escolares con triglicéridos elevados $(19,4 \%)$, HDL-c bajo (44,8\%) y LDL-c elevado $(7,5 \%)$. En cambio, en el distrito de Bellavista-Callao se identificó la mayor prevalencia de obesidad abdominal (25,0\%). Todas estas diferencias fueron significativas $(p<0,01)$.

Entre los universitarios, las prevalencias más altas fueron obesidad abdominal (35\%), HDL bajo $(21,1 \%)$ y sobrepeso/obesidad (20,9\%), como se muestra en la Tabla 3, los del distrito de Trujillo-La Libertad tenían el mayor porcentaje de obesidad abdominal $(42,1 \%)$, siendo estas las únicas diferencias significativas.

En las mujeres de OSB, las prevalencias más elevadas fueron obesidad abdominal (91,8\%), sobrepeso/ obesidad $(73,4 \%)$ y colesterol total elevado $(50,1 \%)$, como se muestra en la tabla 3. Se encontraron diferencias en los porcentajes de hipertensión arterial $(p<0,001)$, siendo mayor la frecuencia para el distrito de Bellavista-Callao (31,7\%) en comparación de Trujillo-La Libertad $(0,9 \%)$. No se encontraron diferencias para la hipercolesterolemia total $(p=0,99)$, hipertrigliceridemia $(p=0,11)$ u obesidad abdominal $(p=0,22)$ según distrito.

\section{PRÁCTICAS SALUDABLES Y NOCIVAS}

Las prácticas saludables menos prevalentes fueron el consumo adecuado de agua, consumo adecuado de frutas y verduras y adecuada actividad física. Mientras que, entre el $20-34 \%$ refirió haber fumado alguna vez en su vida, y entre el 58-84\% refirió haber consumido licor alguna vez en su vida. Detalles adicionales sobre las prevalencias según población se presentan en la Tabla 4. En los escolares no hubo diferencias según

Tabla 3. Prevalencia de enfermedades no transmisibles cardiovasculares y metabólicas en poblaciones provenientes de cuatro distritos del Perú

\begin{tabular}{|c|c|c|c|c|c|c|}
\hline & \multicolumn{2}{|c|}{ Escolares } & \multicolumn{2}{|c|}{ Universitarios } & \multicolumn{2}{|c|}{ Madres OSB } \\
\hline & \multicolumn{2}{|c|}{$\%$ (IC 95\%) } & \multicolumn{2}{|c|}{ \% (IC 95\%) } & \multicolumn{2}{|c|}{$\%$ (IC 95\%) } \\
\hline Sospecha de diabetes & 1,4 & $(0,0-3,3)$ & 1,0 & $(0,0-1,9)$ & 20,3 & $(14,6-25,9)$ \\
\hline Colesterol total elevado & 5,2 & $(2,5-7,8)$ & 11,5 & $(8,4-14,7)$ & 50,1 & $(45,2-55,0)$ \\
\hline Triglicéridos elevados & 7,8 & $(4,6-11,0)$ & 10,3 & $(7,3-13,3)$ & 39,4 & $(34,7-44,2)$ \\
\hline HDL bajo & 30,9 & $(25,3-36,4)$ & 21,1 & $(17,1-25,1)$ & 33,8 & $(29,1-38,4)$ \\
\hline LDL elevado & 3,3 & $(1,1-5,5)$ & 8,5 & $(5,8-11,3)$ & 37,7 & $(32,9-42,4)$ \\
\hline Hipertensión arterial & -- & -- & 1,2 & $(0,1-2,1)$ & 12,8 & $(9,6-16,1)$ \\
\hline Sobrepeso/obesidad & 11,3 & $(7,6-15,0)$ & 20,9 & $(17,0-24,7)$ & 73,4 & $(69,1-77,6)$ \\
\hline Obesidad abdominal & 14,1 & $(10,1-18,2)$ & 35,0 & $(30,5-39,6)$ & 91,8 & $(89,1-94,4)$ \\
\hline
\end{tabular}


distrito de procedencia para el consumo adecuado de agua $(p=0,30)$, actividad física $(p=0,42)$, consumo de frutas $(p=0,24)$ o respuesta ante conflictos $(p=0,60)$; solo existieron diferencias en la cantidad de sueño adecuado $(p<0,001)$, donde el porcentaje de escolares que duermen menos de 7 horas diarias fue mayor en el distrito de Chaclacayo-Lima (25,3\%) en comparación con los escolares de Trujillo-La Libertad (4,9\%).

En los universitarios, el consumo adecuado de agua fue mayor en Chaclacayo-Lima (69,2\%) en comparación con Bellavista-Callao (24,5\%) siendoestadiferenciasignificativa $(p<0,001)$. La actividad física fue menor en estos distritos $(<20 \%)$ en comparación con Hunter-Arequipa $(40,4 \%)$ o Trujillo-La Libertad (47,4\%). No se encontraron diferencias significativas en el tiempo adecuado de sueño $(p=0,13)$, en la respuesta ante conflictos $(p=0,11)$.

En las mujeres de OSB, las procedentes del distrito de Trujillo-La Libertad presentaron el porcentaje más elevado de consumo adecuado de agua (51,4\%), mientras que fue menor entre las de Bellavista-Callao (25,8\%). El $47,7 \%$ de las procedentes de ChaclacayoLima tenían una inadecuada cantidad de sueño, que fue mayor al porcentaje de los otros distritos $(<40 \%)$. El $45,9 \%$ de las procedentes de Trujillo presentaron una adecuada cantidad de actividad física, en comparación con los otros distritos, donde fue de menos del $20 \%$.

\section{DISCUSIÓN}

Las enfermedades cardiovasculares y metabólicas son frecuentes en la población adulta peruana $(2,4)$; según reportes oficiales los factores de riesgo más frecuentes son el sobrepeso y la obesidad con $51,8 \%$, y la hipercolesterolemia con $19,6 \%{ }^{(23)}$. En este estudio, estos resultados son consistentes con la población adulta incluida, lo que permite comparar y corroborar la solidez de nuestra información. Además, se incluye una población de escolares y otra de universitarios, que son poblaciones más jóvenes y no provenientes de hospitales, en las que los estudios son escasos, siendo ello una fortaleza de nuestra investigación.

Las mayores prevalencias de FRCRM se dan en los distritos de Lima y Bellavista-Callao, que son ciudades peruanas con un mayor índice de urbanización respecto a La Libertad o Arequipa. Este resultado es concordante con otros estudios epidemiológicos que señalan que algunas enfermedades metabólicas, como la obesidad (7) y la hipertensión arterial ${ }^{24)}$, son más prevalentes en ciudades con un mayor índice de urbanización.

El sobrepeso y obesidad son factores de riesgo para el desarrollo de enfermedades cardiovasculares no transmisibles ${ }^{(25)}$, diabetes tipo 2, hipertensión arterial, dislipidemia, cáncer y apnea del sueño (26,27) y son altamente prevalentes en nuestro estudio con énfasis en mujeres adultas. En el reciente estudio de Álvarez et al. (7) donde calcularon la prevalencia de exceso de peso a nivel nacional, en adolescentes de ambos sexos fue de $17,1 \%$ en el área urbana, y en mujeres adultas de $65,8 \%$; resultados que son similares al presente estudio.

La prevalencia de hipertensión en las mujeres de OSB procedentes de Lima y Callao son las más altas identificadas en este estudio, pero consistentes con las prevalencias reportadas para estos departamentos en estudios epidemiológicos a nivel nacional ${ }^{(24)}$. En cambio, las prevalencias que se identificaron para las mujeres de Arequipa y La Libertad es mucho menor, posiblemente por el intervalo de edad donde se encontraba la muestra. Para la población universitaria, la prevalencia identificada es baja, menor a la descrita en Chile donde el 12,8\% presentaba hipertensión (15); en

Tabla 4. Prácticas saludables y nocivas desarrolladas por la población estudiada.

\begin{tabular}{lccc}
\hline & Escolares & Universitarios & Madres OSB \\
\cline { 2 - 4 } & $\%$ (IC 95\%) & $\%$ (IC 95\%) & \% (IC 95\%) \\
\hline Prácticas saludables & & & \\
Consumo adecuado de agua & $34,3(28,9-39,8)$ & $40,1(35,5-44,8)$ & $34,1(29,5-38,8)$ \\
Cantidad adecuada de horas de sueño & $50,5(44,6-56,5)$ & $50,8(46,1-55,6)$ & $52,2(47,3-57,1)$ \\
Actividad física adecuada & $43,4(37,7-49,3)$ & $35,5(30,9-40,0)$ & $29,3(24,9-33,7)$ \\
Consumo adecuado de frutas y verduras & $31,5(26,0-36,9)$ & $23,4(19,4-27,4)$ & $16,9(13,3-20,6)$ \\
Respuesta activa antes conflictos habituales & $60,8(55,0-66,6)$ & $62,2(57,6-66,8)$ & $61,8(57,1-66,5)$ \\
Prácticas nocivas* & & & \\
Alguna vez fumó & $20,4(15,6-25,1)$ & $30,5(26,1-34,9)$ & $33,9(29,3-38,5)$ \\
Fuma solo en fiestas o reuniones & $7,5(4,3-10,6)$ & $12,7(9,5-15,8)$ & $12,1(8,9-15,3)$ \\
Alguna vez consumió licor & $58,4(52,6-64,2)$ & $71,4(67,1-75,7)$ & $84,4(80,9-87,9)$ \\
Consumió licor el último mes & $36,4(28,4-44,4)$ & $50,4(44,4-56,3)$ & $32,1(27,1-37,2)$ \\
Consumió licor la última semana & $30,8(21,1-40,4)$ & $34,4(28,2-40,5)$ & $23,2(17,9-28,6)$ \\
\hline
\end{tabular}

* Las categorías son independientes unas de otras 
cambio, la prevalencia de sospecha de diabetes sí es consistente con el mismo estudio, donde el 1,6\% tenía glicemia alterada y no habían casos de diabetes. Esto guarda relación con sus estilos de vida relacionados con el hábito de fumar, el consumo de alcohol y la vida sedentaria de la población chilena estudiada ${ }^{(15)}$.

Este estudio tiene limitaciones, básicamente al no realizar dos mediciones serológicas para confirmar el diagnóstico de diabetes, sin embargo, los pacientes fueron derivados para su evaluación y manejo respectivo. Asimismo, la selección de los sujetos obedeció a personas agrupadas en lugares seleccionados que compartirían similares características socioeconómicas, dado que era la población objetivo para la intervención.

Los FRCVM son más frecuentes en los grupos de mayor edad y en los distritos de Lima y el Callao, donde su prevalencia es alta. En los grupos de escolares y universitarios no son frecuentes, sin embargo, un porcentaje importante presenta problemas de sobrepeso y obesidad, en particular la obesidad abdominal; que según estudios previos está altamente asociado con el desarrollo de FRCRM. Por lo que se recomienda la intervención temprana en la promoción de estilos de vida saludable con énfasis en hábitos de alimentación saludable y mayor actividad física en cada etapa de vida, en particular en los escolares y adolescentes, para así reducir la incidencia de FRCRM en nuestra población.

Los resultados del presente estudio permitirán orientar las intervenciones para reducir los factores de riesgo de estas enfermedades, dado que constituye la línea de base de la intervención "ADELANTE".

Fuentes de financiamiento: colaboración entre ADRA Perú e INS, con contribución financiera de la Unión Europea.

Agradecimiento: al Méd. Charles A. Huamaní Saldaña por su apoyo en el análisis e interpretación de datos y la redacción del artículo.

Contribuciones de autoría: ENR, CHP, RTL Y MYE han participado en la concepción de la investigación, diseño, su ejecución y recolección de datos; además en la concepción del artículo y su redacción. Además ENR realizó la sistematización, al análisis de datos y retroalimentación para la aprobación de la versión final del artículo. VSM y GSL contribuyeron en el diseño del proyecto de la investigación. Además GSL participó en la recolección y sistematización de datos. SEL, participó en la revisión del artículo y brindó facilidades para su publicación.

\section{REFERENCIAS BIBLIOGRÁFICAS}

1. World Health Organization. Global status report on noncommunicable diseases 2010. Geneva: WHO; 2011.

2. Organizacion Panamericana de la Salud, Ministerio de Salud. Situación de las enfermedades no transmisibles en el Perú. Lima: OPS; 2003.

3. Huicho L, Trelles M, Gonzales F, Mendoza W, Miranda J. Mortality profiles in a country facing epidemiological transition: an analysis of registered data. BMC Public Health. 2009 Feb 2;9:47. doi: 10.1186/14712458-9-47.

4. Velásquez A. La carga de enfermedad y lesiones en el Perú y las prioridades del plan esencial de aseguramiento universal. Rev Peru Med Exp Salud Publica. 2009 Abr-Jun;26(2):222-31.

5. Ministerio de Salud. Dirección General de Epidemiología. Estudio de carga de enfermedad en la provincia de Lima y región del Callao [Internet]. Lima: MINSA; 2010. Disponible en: http:// www.dge.gob.pe/publicaciones/pub_ asis/asis28.pdf

6. Miranda JJ, Wells JCK, Smeeth L. Transiciones en contexto: Hallazgos vinculados a migración rural-urbana y enfermedades no transmisibles en Perú. Rev Per Med Exp Salud Publica. 2012 Jul-Set;29(3):366-72.

7. Álvarez-Dongo D, Sánchez-Abanto J, Gómez-Guizado G, Tarqui-Mamani C. Sobrepeso y obesidad: prevalencia y determinantes sociales del exceso de peso en la población peruana (20092010). Rev Peru Med Exp Salud Publica. 2012 Jul-Set;29(3):303-13.

8. Pajuelo J, Bernui I, Nolberto V, Peña A, Zevillanos L. Síndrome metabólico en adolescentes con sobrepeso y obesidad. An Fac Med. 2007 AbrJun;68(2):143-9.

9. Sáez Y, Bernui I. Prevalencia de factores de riesgo cardiovascular en adolescentes de instituciones educativas. An Fac Med. 2009 Dic;70(4):259-65.

10. Ministerio de Salud. Dirección General de Promoción de la Salud [Internet]. Lima: DGPS; 2003 [citado el 30 de diciembre del 2013]. Disponible en: http://www.minsa.gob.pe/dgps/ promocion/dgps.html

11. Ministerio de Salud. Dirección General de Promoción de la Salud. Dirección ejecutiva de promoción de vida sana [Internet]. Lima: MINSA; 2012 [citado el 30 de Diciembre del 2013]. Disponible en: ftp://ftp2.minsa.gob. pe/descargas/dgps/documentos/ brochure_dgps.pdf

12. Ekelund U, Ong KK, Linné Y, Neovius M, Brage S, Dunger DB, et al. Association of weight gain in infancy and early childhood with metabolic risk in young adults. J Clin Endocrinol Metab. 2007 Jan;92(1):98-103.

13. Adventist Development and Relief Agency Peru. ADELANTE: Estrategia preventiva-promocional para disminuir las enfermedades no transmisibles en adolescentes, jóvenes y adultos [Internet]. Lima: ADRA; 2011 [citado el 26 de diciembre del 2013]. Disponible en: http://www.adra.org. pe/en/web/secciones../proyecto_ detalle.php?idlineaaccion=9\&idproye cto $=11$

14. Bejarano I, Dipierri J, Alfaro E, Quispe Y, Cabrera G. Evolución de la prevalencia de sobrepeso, obesidad y desnutrición en escolares de San Salvador de Jujuy. Arch Argent Pediatr. 2005;103(2):101-9.

15. Palomo IF, Torres GI, Alarcón MA, Maragaño PJ, Leiva E, Mujica 
V. Alta prevalencia de factores de riesgo cardiovascular clásicos en una población de estudiantes universitarios de la región centro-sur de Chile. Rev Esp Cardiol. 2006;59(11):1099-105.

16. Seclén J, Miranda M, Sánchez J, de la Cruz L, Chávez H; Ministerio de Salud, Instituto Nacional de Salud. Comportamiento de indicadores de nutrición en el Perú-Monitoreo nacional de indicadores nutricionales Perú 2002-2004. Lima: MINSA-INS; 2007.

17. Chobanian AV, Bakris GL, Black HR, Cushman WC, Green LA, Izzo JL Jr., et al. Seventh report of the Joint National Committee on Prevention, Detection, Evaluation, and Treatment of High Blood Pressure. Hypertension 2003 Dec;42(6):1206-52.

18. Organización Mundial de la Salud. Obesidad y sobrepeso [Internet]. Ginebra: OMS; 2014 [citado el 26 de diciembre del 2013). Disponible en: http://www.who.int/mediacentre/ factsheets/fs311/es/

19. Grundy SM, Cleeman JI, Daniels SR, Donato KA, Eckel RH, Franklin BA, et al. Diagnosis and management of the metabolic syndrome: an American Heart Association/National Heart, Lung, and Blood Institute Scientific Statement. Circulation. 2005 Oct 25;112(17):2735-52.
20. Expert Committee on the Diagnosis and Classification of Diabetes Mellitus. Report of the expert committee on the diagnosis and classification of diabetes mellitus. Diabetes Care. 2003;26 Suppl $1: S 5-20$.

21. Expert Panel on Detection, Evaluation, and Treatment of High Blood Cholesterol in Adults. Executive Summary of The Third Report of The National Cholesterol Education Program (NCEP) Expert Panel on Detection, Evaluation, and Treatment of High Blood Cholesterol In Adults (Adult Treatment Panel III). JAMA. 2001 May 16;285(19):2486-97.

22. Chile; Ministerio de Salud. Subsecretaría de Salud Pública. División de Planificación Sanitaria. II Encuesta de Calidad de Vida y Salud Chile, 2006 [Internet]. Santiago de Chile: MINSA; 2006 [citadoel27 de diciembre del2013]. Disponible en: http://epi.minsal.cl/epi/ html/sdesalud/calidaddevida2006/ Informe $\% 20$ Final $\% 20$ Encuesta $\% 20$ de\%20Calidad\%20de\%20Vida $\% 20$ y\%20Salud\%202006.pdf

23. Perú, Ministerio de Salud. Instituto Nacional de Salud, Centro Nacional de Alimentación y Nutrición. Encuesta Nacional de Indicadores Nutricionales, Bioquímicos, Socioeconómicos y Culturales relacionados con las enfermedades crónicas degenerativas. Lima: INS-CENAN; 2006.
24. Agusti R. Epidemiología de la Hipertensión Arterial en el Perú. Acta Med Peruana. 2006 MayAgos;23(2):69-75.

25. Pajuelo J, Sánchez J. El síndrome metabólico en adultos, en el Perú. An Fac Med. 2007;68(1):38-46.

26. Ford ES, Giles WH, Dietz WH. Prevalence of the metabolic syndrome among US adults: findings from the third National Health and Nutrition Examination Survey. JAMA. 2002 Jan 16;287(3):356-9.

27. Márquez-Sandoval F, Macedo-Ojeda G, Viramontes-Hörner D, Fernández Ballart JD, Salas Salvadó J, Vizmanos B. The prevalence of metabolic syndrome in Latin America: a systematic review. Public Health Nutr. 2011 Oct;14(10):1702-13. doi: 10.1017/ S1368980010003320.

Correspondencia: María Eloisa Núñez Robles Dirección: Calle Palma Real 115 Urb. Parque de Monterrico - Camacho, La Molina, Lima 12.

Teléfono: 990903955

Correo electrónico: enunezr2007@gmail.com

\section{Consulte la versión electrónica de la} Revista Peruana de Medicina Experimental y Salud Pública en

\section{www.pubmed.gov}

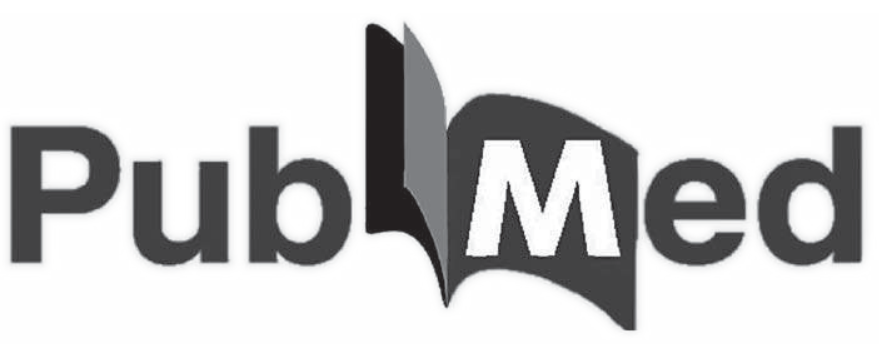

